\title{
High-Throughput Docking and Molecular Dynamics Simulations towards the Identification of Potential Inhibitors against Human Coagulation Factor XIIa
}

\author{
Dongfang Xu, ${ }^{1}$ Guangpu Xue, ${ }^{2}$ Bangya Peng, ${ }^{3}$ Zanjie Feng, ${ }^{3}$ Hongling Lu $\left(\mathbb{D},{ }^{3}\right.$ \\ and Lihu Gong $\mathbb{D}^{3}$ \\ ${ }^{1}$ Library of Zunyi Medical University, China \\ ${ }^{2}$ Institute of Chemistry and Biochemistry, Freie Universität Berlin, Germany \\ ${ }^{3}$ Department of Biochemistry, Zunyi Medical University, China \\ Correspondence should be addressed to Hongling Lu; luhl@zmu.edu.cn and Lihu Gong; 240239973@qq.com
}

Received 12 January 2020; Revised 22 March 2020; Accepted 7 April 2020; Published 22 May 2020

Academic Editor: John Mitchell

Copyright (C) 2020 Dongfang Xu et al. This is an open access article distributed under the Creative Commons Attribution License, which permits unrestricted use, distribution, and reproduction in any medium, provided the original work is properly cited.

\begin{abstract}
Human coagulation factor XIIa (FXIIa) is a trypsin-like serine protease that is involved in pathologic thrombosis. As a potential target for designing safe anticoagulants, FXIIa has received a great deal of interest in recent years. In the present study, we employed virtual high-throughput screening of 500,064 compounds within Enamine database to acquire the most potential inhibitors of FXIIa. Subsequently, 18 compounds with significant binding energy (from -65.195 to $-15.726 \mathrm{kcal} / \mathrm{mol}$ ) were selected, and their ADMET properties were predicted to select representative inhibitors. Three compounds (Z1225120358, Z432246974, and Z146790068) exhibited excellent binding affinity and druggability. MD simulation for FXIIa-ligand complexes was carried out to reveal the stability and inhibition mechanism of these three compounds. Through the inhibition of activated factor XIIa assay, we tested the activity of five compounds Z1225120358, Z432246974, Z45287215, Z30974175, and Z146790068, with pIC50 values of $9.3 * 10^{-7}, 3.0 * 10^{-5}, 7.8 * 10^{-7}, 8.7 * 10^{-7}$, and $1.3 * 10^{-6} \mathrm{M}$, respectively; the AMDET properties of Z45287215 and Z30974175 show not well but have better inhibition activity. We also found that compounds Z1225120358, Z45287215, Z30974175, and Z146790068 could be more inhibition of FXIIa than Z432246974. Collectively, compounds Z1225120358, Z45287215, Z30974175, and Z146790068 were anticipated to be promising drug candidates for inhibition of FXIIa.
\end{abstract}

\section{Introduction}

Human coagulation factor XIIa (FXIIa) is an important component involved in the initiation of the intrinsic pathway of the coagulation cascade [1]. The intrinsic coagulation cascade is initiated by contact activation in reactions involving high-molecular-weight kininogen (HMWK) and prekallikrein (PPK) [2]. The contact system can be activated by diverse negatively charged polymers, including kaolin, nucleic acids [3], and collagen [4]. Contacting with polyanions, FXII undergoes a conformational change and converts into active form $\alpha$-FXIIa [5]. Essentially, kallikrein cleaves the FXII Arg353-Val354 peptide bond, generating $\alpha$-FXIIa, which consists of a N-terminal heavy chain with a molecular weight of $50 \mathrm{kDa}$ and a Cys340-Cys467 disulfide bridge-connected C-terminal light chain with a molecular weight of $28 \mathrm{kDa}$. The $\mathrm{N}$-terminal heavy chain comprises a contact-binding domain, while the C-terminal light chain comprises a catalytic center. Once a small amount of $\alpha$-FXIIa generate, it can further cleave prekallikrein to generate kallikrein. $\alpha$-FXIIa and kallikrein then form a synergetic system to amplify their production. $\alpha$-FXIIa activates factor XI to factor XIa, which contributes to plasma coagulation [6]. Subsequent cleavage of $\alpha$-FXIIa results in loss of the heavy chain and generation of the isolated protease domain termed $\beta$ FXIIa, consisting of a nine amino acid peptide heavy chain remnant disulfide bonded to the protease domain $[5,7-9]$.

Inhibition of FXII activity is an attractive approach for the treatment and prevention of thrombotic diseases [10]. Previous studies show that FXII-deficient mice are protected 
from arterial thrombosis, ischemic stroke, and deep vein thrombosis while maintaining normal hemostasis $[11,12]$, suggesting that FXII is critically involved in pathologic thrombus formation but is dispensable for hemostasis. For more than five decades, it has been known that deficiency of coagulation factor XII is not associated with increased spontaneous or injury-related bleeding complications [13]. Patients that are deficient in FXII do not suffer from abnormal bleeding even during major surgical procedures [14]. This observation and the limited role of FXII in hemostasis raised the prospect that inhibiting FXIIa could offer an antithrombotic therapy with a low bleeding risk [15].

In recent years, inhibitors for FXIIa have been generated and described in various preclinical models in vitro or in vivo [16]. These agents include monoclonal antibodies $[13,17]$, natural peptide or protein inhibitors $[18,19]$, small molecule inhibitors [12, 20], RNA aptamer [21], small interfering RNA [22], and antisense oligonucleotide [23]. Currently available antithrombotic agents such as heparin, warfarin, and antiplatelet therapy can cause serious bleeding complication, because they target components of the blood-clotting mechanism such as thrombin, FVIIa, FIXa, FXa, and FXIa [24]. Therefore, it is necessary to design new FXIIa inhibitors and optimize them into therapeutic agents, which have high inhibitory activity against FXIIa without increasing the bleeding risk and exist stably in human plasma.

Structure-based drug design relies on the knowledge of the three-dimensional structure of the biomolecular target. It is extensively applied to find potent and specific inhibitors against a particular drug [25-27]. This study reports the identification and validation of novel FXIIa inhibitors using high-throughput screening (HTS), docking, and molecular dynamics (MD) simulation-based approaches. Finally, MD simulations were performed on FXIIa, FXIIaZ1225120358 complex, FXIIa-Z432246974 complex, and FXIIa-Z146790068 complex to get deeper insights into the binding mechanism of FXIIa to the selected ligands.

\section{Materials and Methods}

2.1. Materials. The study was carried out on HP Workstation with $3.5 \mathrm{GHz}$ processor, $8 \mathrm{~GB}$ RAM, and $1 \mathrm{~TB}$ hard drive running in Windows operating system, high speed internet (broadband) connection, and uninterrupted and stabilized power supply. Bioinformatics software: Discovery Studio, GraphPad Prism software, and online resources, like NCBI, RCSB, and Enamine database, were used in this study.

Full length-activated FXIIa (a-FXIIa) were obtained from Enzyme Research Laboratories (Swansea, UK). Commercial compounds were obtained from Enamine (KIEV, Ukraine). S2302 (a chromogenic substrate peptide mimic) was obtained from Chromogenix (Epsom, UK).

2.2. Databases Used for Screening. Enamine Library, a free database of commercially available compounds, was used to perform virtual screening. This approach allowed the identification of 500,064 compounds, which contains kinase inhibitor library.
2.3. High-Throughput Screening. The docking studies were based on the available crystal structure of FXIIa (PDB code: 6B77). Water molecules and heteroatom including ligands were removed. Compounds obtained from the Enamine database were docked into the FXIIa protein active site. HTS was then performed using the Libdock method, which has been incorporated into Discovery Studio 2018 (Discovery Studio User Manual).

Before docking, the receptor structure was typed with CHARMM force field [28], hydrogen atoms were added, and $\mathrm{pH}$ of the protein was adjusted to almost neutral 7.4 using protein preparation module. All ligands were typed with CHARMM force field and minimized by using the smart minimizer minimization algorithm of Discovery Studio 2018, which contains 1000 steps of steepest descent with a RMS gradient tolerance of 3 and conjugate gradient minimization. The ten top-scoring conformations of every ligand were then saved at the end of calculation. For all compounds, the docked structures with the highest scores were analyzed to identify the molecules. Finally, the complexes of 18 top-ranking compounds with $6 \mathrm{~B} 77$ were subjected to free energy calculations.

2.4. Binding Free Energy Calculation. To estimate the binding energy between a receptor and a ligand, we calculated the average binding energy across a set of related poses [29]. The binding energy is calculated using the following equation: Energy $y_{\text {Binding }}=$ Energy $_{\text {Complex }}-$ Energy $_{\text {Ligand }}-$ Energy $_{\text {Receptor }}$. The Binding Free Energy Calculation was carried out using the Discovery Studio 2018 program.

2.5. ADMET Prediction. Binding affinities based on binding energy, top 18 hits were selected which showed a significantly higher binding affinity towards the binding pocket of FXIIa. All these compounds were further subjected to ADMET prediction to get drug-like molecules. As a successful small molecular drug, it should not only be active against a target but also possess appropriate ADMET properties. ADMET properties play vital roles in the discovery and development of small molecule drugs [30]. We have further performed ADMET Descriptors and Toxicity Prediction methods in Discovery Studio 2018. These ADMET modules include aqueous solubility, BBB penetration, cytochrome $\mathrm{P} 450$ (CYP450) 2D6 inhibition, hepatotoxicity, human intestinal absorption (HIA), and plasma protein binding. The data for establishing these modules are derived from a large number of literature reports and experimental data, and these models have been widely validated (Discovery Studio User Manual).

2.6. Molecular Dynamics Simulations. MD simulations were performed on FXIIa, FXIIa-Z1225120358 complex, FXIIaZ432246974 complex, and FXIIa-Z146790068 complex. All simulations were carried out with Discovery Studio 2018. The CHARMM force field was applied to both protein and small molecules. The protein-ligand systems were solvated for the simulation, adding enough water molecules to allow the protein to interact with the solvent naturally. The protein should be solvated in a water box, which allows the simulation to run using periodic boundary conditions to avoid surface artifacts [31]. 
TABLE 1: Compounds selected as potential FXIIa inhibitors by HTS filter and Binding Free Energy Calculations.

\begin{tabular}{|c|c|c|c|c|c|c|c|}
\hline No. & Compound id & MW & Binding energy $(\mathrm{kcal} / \mathrm{mol})$ & $\mathrm{Alog} \mathrm{P}$ & H-bond donor & H-bond acceptor & Chemical structure \\
\hline 1 & Z45287215 & 310.372 & -65.1947 & 3.4685 & 1 & 1 & \\
\hline 2 & Z30974175 & 424.303 & -50.0193 & 3.6279 & 2 & 3 & \\
\hline 3 & Z1225120358 & 317.296 & -39.8838 & 2.6222 & 1 & 4 & \\
\hline 4 & Z53058673 & 330.387 & -39.6591 & 3.0745 & 1 & 2 & \\
\hline 5 & Z53059201 & 341.414 & -38.4803 & 2.9696 & 1 & 2 & \\
\hline 6 & Z432246974 & 387.45 & -36.396 & 2.8377 & 2 & 4 & \\
\hline 7 & Z56867305 & 300.314 & -34.1774 & 3.2588 & 1 & 2 & \\
\hline 8 & Z223449194 & 313.33 & -30.3052 & 3.3693 & 1 & 2 & \\
\hline 9 & Z818810338 & 430.464 & -26.6265 & 3.736 & 1 & 4 & \\
\hline 10 & Z146790068 & 353.397 & -25.4344 & 2.6916 & 0 & 3 & \\
\hline
\end{tabular}


TABLe 1: Continued.

\begin{tabular}{|c|c|c|c|c|c|c|c|}
\hline No. & Compound id & MW & Binding energy $(\mathrm{kcal} / \mathrm{mol})$ & $\mathrm{A} \log \mathrm{P}$ & H-bond donor & H-bond acceptor & Chemical structure \\
\hline 11 & Z53059185 & 341.414 & -23.701 & 3.1847 & 1 & 2 & \\
\hline 12 & Z19630209 & 393.399 & -22.6212 & 1.5979 & 1 & 4 & \\
\hline 13 & Z603981096 & 348.406 & -20.2983 & 3.8445 & 2 & 3 & \\
\hline 14 & Z53058577 & 344.414 & -19.9549 & 3.3227 & 1 & 2 & \\
\hline 15 & Z132701382 & 344.414 & -17.8038 & 3.5587 & 1 & 2 & \\
\hline 16 & Z603981096 & 348.406 & -17.7091 & 3.8445 & 2 & 3 & \\
\hline 17 & Z914249910 & 322.412 & -17.1128 & 2.6614 & 1 & 3 & \\
\hline 18 & Z1392999050 & 360.361 & -15.7256 & 3.2774 & 1 & 4 & \\
\hline
\end{tabular}

Initially, the system underwent 1000 steps of steepest descent minimization and 2000 steps of conjugate gradient minimization. The energy minimization step was followed by heating, equilibration, and production. The whole system was heated from an initial temperature of $50 \mathrm{~K}$ to $300 \mathrm{~K}$ in 4 ps without restraint. The equilibration was run in $300 \mathrm{~K}$ for $20 \mathrm{ps}$ without restraint. The production was run in $300 \mathrm{~K}$ for a time of $200 \mathrm{ps}$ with typed NPT. The total simulation time over the heating, equilibration, and production steps is 224 ps. The electrostatic parameter was set to automatic, which automatically recognizes the periodic environment and sets the electrostatic calculation to Particle Mesh Ewald (PME). RMSD and RMSF were computed for the entire protein molecule using the starting structure as reference. Hydrogen bonding between the FXIIa protein and the compound was monitored and analyzed over the course of the simulation [32].

2.7. FXIIa Enzymatic Assay. The enzymatic activity of aFXIIa was measured by monitoring the amount of pNA chromophore released from substrate H-D-Pro-Phe-ArgpNA (S-2302). Assays were performed in a $100 \mu \mathrm{L}$ volume at $33^{\circ} \mathrm{C}$ in 96 -well plates in a PerkinElmer (Seer Green, UK) Envision plate reader, and pNA release was followed over a period of $6 \mathrm{~h}$ by reading the absorbance at $405 \mathrm{~nm}$. Absorbance values were converted to pNA concentrations by comparison with a standard curve obtained under exactly the same instrument conditions. All absorbance values were within the linear measurement range of the instrument. Initial rates were calculated on the basis of the first $30 \mathrm{~min}$ of 
TABLE 2: The ADMET prediction results of inhibitors.

\begin{tabular}{|c|c|c|c|c|c|c|c|c|c|c|c|c|}
\hline No. & $\begin{array}{c}\text { Compound } \\
\text { id }\end{array}$ & Solubility & $\begin{array}{c}\text { Absorption } \\
\text { level }\end{array}$ & $\begin{array}{l}\text { CYP2D6 } \\
\text { inhibitor }\end{array}$ & $\mathrm{BBB}$ & $\begin{array}{l}\text { BBB } \\
\text { LEVEL }\end{array}$ & PPB & РPB & PSA & ogP98 & $\begin{array}{c}\text { Ames } \\
\text { mutagenicity }\end{array}$ & Carcinogenicity \\
\hline 1 & Z45287215 & -5.288 & 0 & True & 0.363 & 1 & -3.123 & False & 45.166 & 3.984 & Nonmutagen & Non \\
\hline 2 & Z30974175 & -5.754 & 0 & False & -0.899 & 3 & -3.97672 & False & 97.957 & 2.604 & Mutagen & Yes \\
\hline 3 & Z1225120358 & -3.705 & 0 & False & -0.503 & 2 & 0.170651 & True & 73.237 & 2.619 & Nonmutagen & Non \\
\hline 4 & Z53058673 & -5.208 & 0 & False & 0.113 & 1 & -8.74339 & False & 57.72 & 3.817 & Nonmutagen & Yes \\
\hline 5 & Z53059201 & -4.92 & 0 & False & 0.03 & 1 & -11.4012 & False & 56.427 & 3.485 & Nonmutagen & Non \\
\hline 6 & Z432246974 & -3.282 & 0 & False & -1.139 & 3 & 4.41143 & True & 98.176 & 1.84 & Nonmutagen & Non \\
\hline 7 & Z56867305 & -4.232 & 0 & False & -0.442 & 2 & 3.37623 & True & 81.864 & 3.258 & Mutagen & Yes \\
\hline 8 & Z223449194 & -5.198 & 0 & False & -0.329 & 2 & 5.26529 & True & 84.195 & 3.744 & Mutagen & Non \\
\hline 9 & Z818810338 & -4.591 & 0 & False & -0.634 & 3 & -0.75978 & True & 94.066 & 3.264 & Nonmutagen & Yes \\
\hline 10 & Z146790068 & -3.545 & 0 & False & -0.206 & 2 & 1.81081 & True & 55.87 & 2.691 & Nonmutagen & Non \\
\hline 11 & Z53059185 & -5.107 & 0 & False & 0.097 & 1 & -9.41512 & False & 56.427 & 3.7 & Nonmutagen & Non \\
\hline 12 & Z19630209 & -2.668 & 0 & False & N/A & 4 & -5.10215 & False & 117.504 & 1.497 & Nonmutagen & Yes \\
\hline 13 & Z603981096 & -4.033 & 0 & False & -0.243 & 2 & -4.36726 & False & 70.888 & 3.34 & Nonmutagen & Yes \\
\hline 14 & Z53058577 & -5.313 & 0 & False & 0.189 & 1 & -8.47245 & False & 57.72 & 4.066 & Nonmutagen & Yes \\
\hline 15 & Z132701382 & -5.703 & 0 & False & 0.262 & 1 & -8.8002 & False & 57.72 & 4.302 & Nonmutagen & Yes \\
\hline 16 & Z603981096 & -4.033 & 0 & False & -0.243 & 2 & -4.36726 & False & 70.888 & 3.34 & Nonmutagen & Yes \\
\hline 17 & Z914249910 & -3.283 & 0 & False & -0.587 & 3 & -2.42367 & False & 79.333 & 2.661 & Nonmutagen & Non \\
\hline 18 & Z1392999050 & -4.152 & 0 & False & -0.263 & 2 & 4.96262 & True & 70.906 & 3.277 & Nonmutagen & Yes \\
\hline
\end{tabular}

incubation. To assay inhibitors, concentrations of five compounds (Z1225120358, Z45287215, Z30974175, Z432246974, and $Z 146790068$ ) between $10^{-8} \mathrm{M}$ and $10^{-3} \mathrm{M}$ were incubated with $200 \mu \mathrm{M}$ substrate peptide, the reaction was initiated by addition of $10 \mathrm{nM}$ a-FXIIa, enzymatic activity was measured as described above, and IC 50 values were determined by nonlinear regression analysis (GraphPad Prism, version 8.0.2.263) using the $\log$ [inhibitor] versus response variable slope algorithm with a bottom constraint.

\section{Result and Discussion}

HTS followed by docking and MD simulation were used to find the potential inhibitors of human FXIIa. A total of 500,064 compounds were screened with the structure of FXIIa, and top-ranked 18 molecules with binding affinity to FXIIa $(\Delta \mathrm{G} \leq-10 \mathrm{kcal} / \mathrm{mol})$ were selected for further analysis (Table 1). All novel hits were accurately fitted within the active site of FXIIa and were further evaluated with druggability parameters.

3.1. High-Throughput Screening and Binding Free Energy Calculation. 500,064 compounds from Enamine databases were virtually screened onto the structure of FXIIa. The structures of the 18 compounds were successfully docked with a binding energy range from $-65.195 \mathrm{kcal} / \mathrm{mol}$ to $-15.726 \mathrm{kcal} /$ mol [33]. Compound Z45287215 showed the highest binding affinity of $-65.195 \mathrm{kcal} / \mathrm{mol}$. The result of this screening is listed in Table 1, and the property contained hydrogen bond donors, hydrogen bond acceptors, binding energy, AlogP, and molecular weight (MW).
3.2. Analysis of the ADMET Prediction. ADMET properties have become one of the most key issues to assess the efficacies or risks of small molecular compounds in biology [34]. The AMDET properties of small molecular compounds could be predicted by integrally analyzing their physicochemical properties, such as molecular weight (MW), polar surface area (PSA), lipophilicity (clogP), and aqueous solubility $(\log S)$, which are directly correlated with properties of drug molecules like absorption and bioavailability [35]. We applied the Discovery Studio ADMET tool to predict the pharmacokinetic profiles of compounds (Table 2).

The blood brain barrier penetration ability of a compound is another equally important parameter for a drug molecule [36]. We found that almost all compounds in Enamine Library are high penetrative except compounds Z30974175, Z432246974, Z818810338, and Z914249910. Human intestinal absorption is given by four prediction levels: "0" (good), “1” (moderate), "2" (poor), and “ 3 " (very poor). The absorption levels of all the compounds are all "0," showing a satisfactory level of absorption. The classification whether a compound is highly bound ( $\geq 90 \%$ bound) to plasma proteins using the cutoff Bayesian score of -2.209 , the results indicate that compounds Z1225120358, Z432246974, Z56867305, Z818810338, Z146790068, and Z1392999050 show strong binding affinity. Almost all compounds are unable to inhibit the CYP450 enzyme activity except compound Z45287215.

All compounds in this study are noncarcinogenic and nonmutagenic except Z30974175, Z53058673, Z56867305, Z223449194, Z818810338, Z19630209, Z603981096, Z53058577, Z132701382, Z603981096, and Z1392999050. Calculated ADME values and other structural properties of 


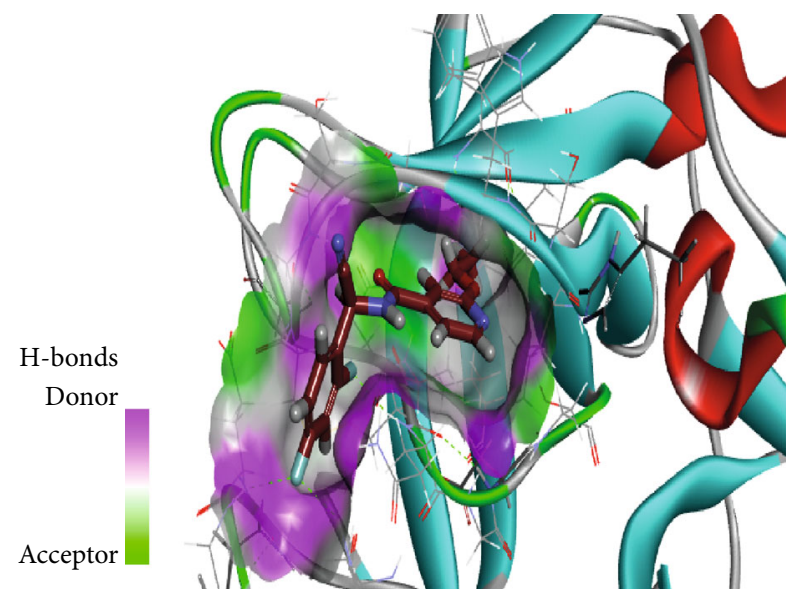

(a)

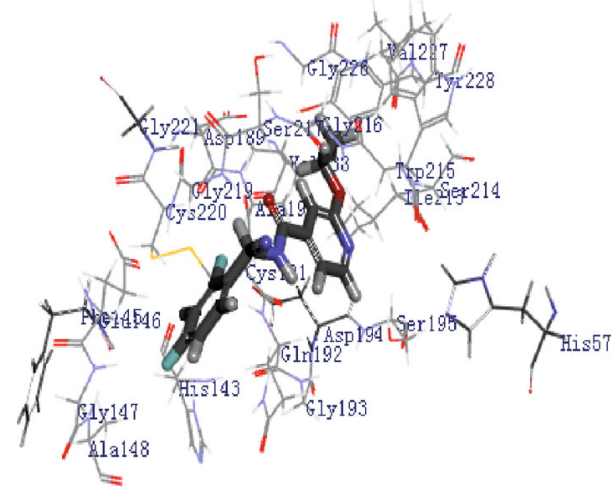

(b)

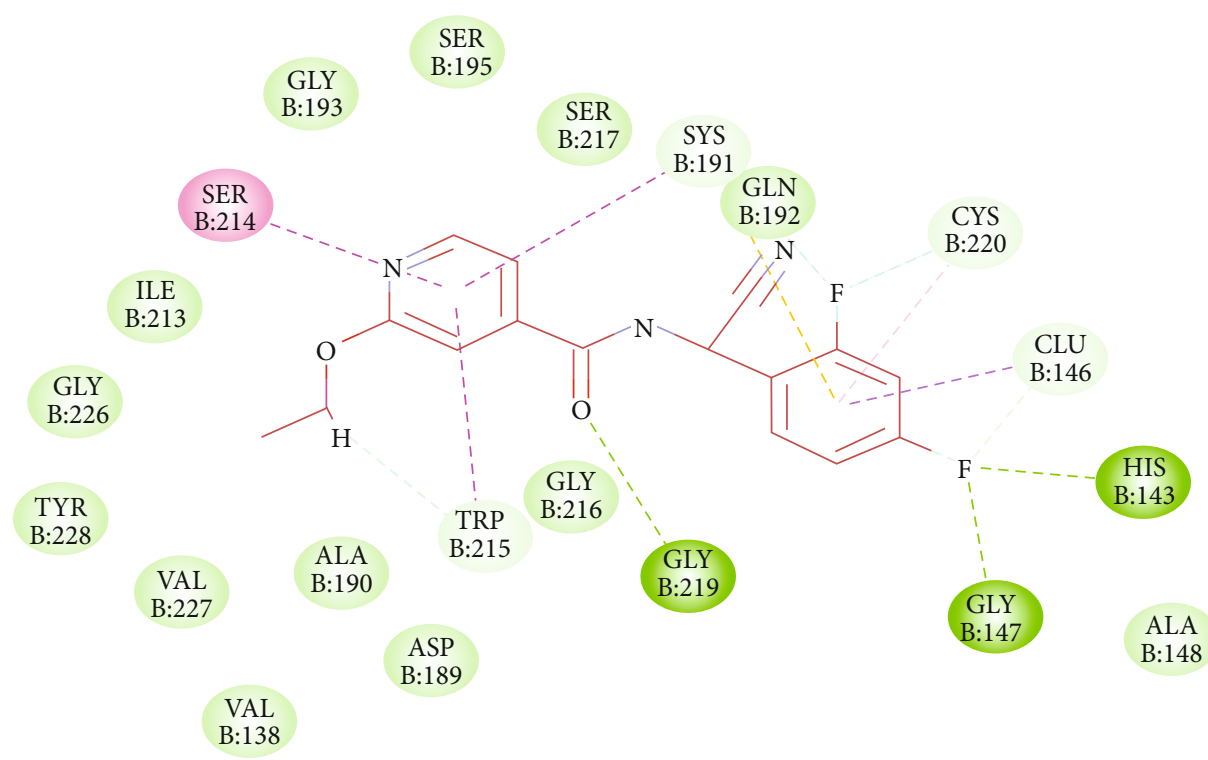

Interactions

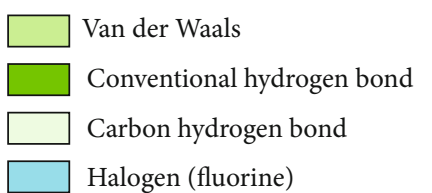

Halogen (fluorine)

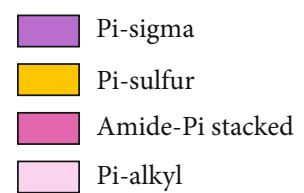

(c)

FIgURe 1: Binding mode of Z1225120358 to the FXIIa. (a) Overall structure of FXIIa-Z1225120358 complex. Protein structure was shown in cartoon and ligand in sticks. Showing the binding site residue surface around the ligand. Displaying the H-bond donor (purple) and acceptor (green) area. (b) Interactions between Z1225120358 (sticks) and FXIIa residues (sticks). (c) 2D diagram of FXIIa interactions to compound Z1225120358.

the FXIIa bound ligands including carcinogenicity mutagenicity are listed in Table 2.

3.3. Structure Analysis. The FXIIa specificity pocket bordered by segments Ile213-Cys220, Asp189-Ser195, Pro225-Thr229, and disulfide bond Cys191-Cys220 is practically identical to that of other active trypsin-like serine proteases. According to the ADMET properties of all compounds, we found that compounds Z1225120358, Z432246974, and Z146790068 have the highest binding affinity towards FXIIa. The AMDET properties of Z45287215 show not well but have the highest binding affinity of $-65.195 \mathrm{kcal} / \mathrm{mol}$.

Structure analysis shows that Z1225120358 binds to the active site cavity of the FXIIa. The estimated binding affinity is significantly high $(-39.884 \mathrm{kcal} / \mathrm{M})$ (Figure $1(\mathrm{a}))$. Three hydrogen bond interactions were observed (His143, Gly147, and Gly219) for Z1225120358 to FXIIa, as well as many van der Waals interactions offered by Asp189, Ala190, Gln192, Ile213, Gly216, Ser217, Gly226, Val227, and Tyr228 (Figures 1(b) and 1(c)). The complex of FXIIa-Z1225120358 


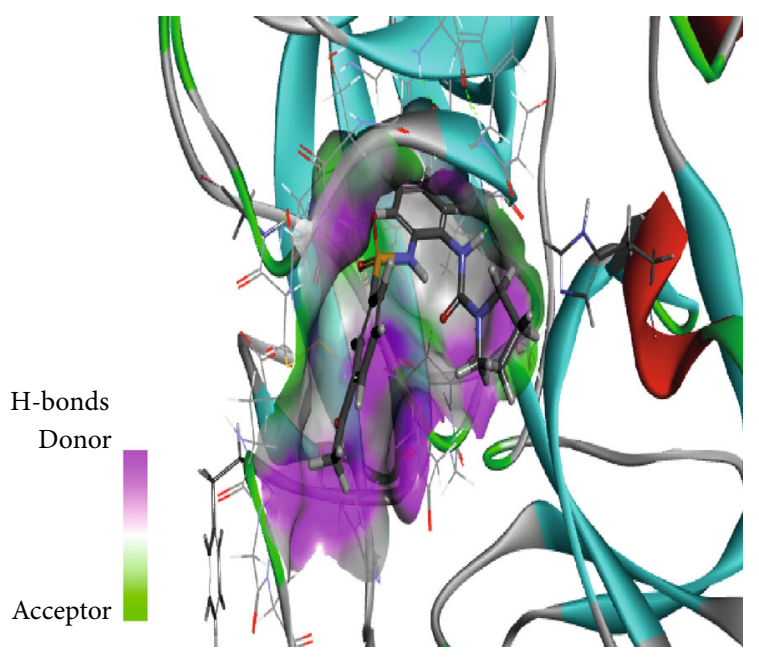

(a)

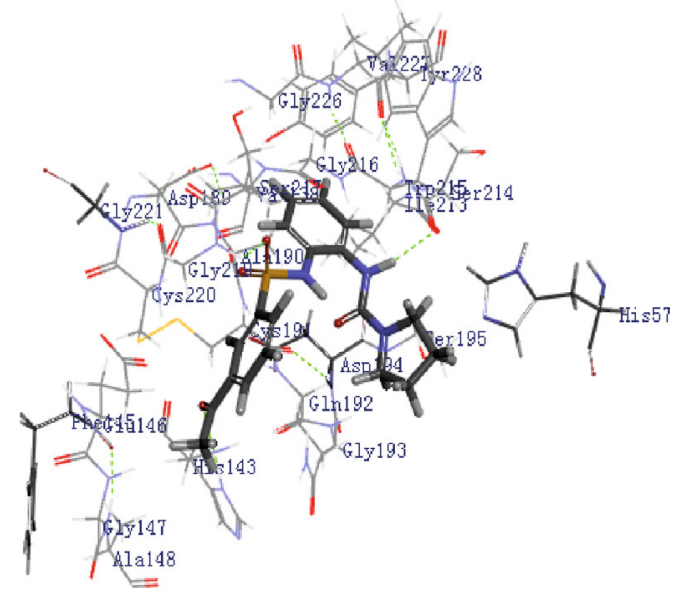

(b)

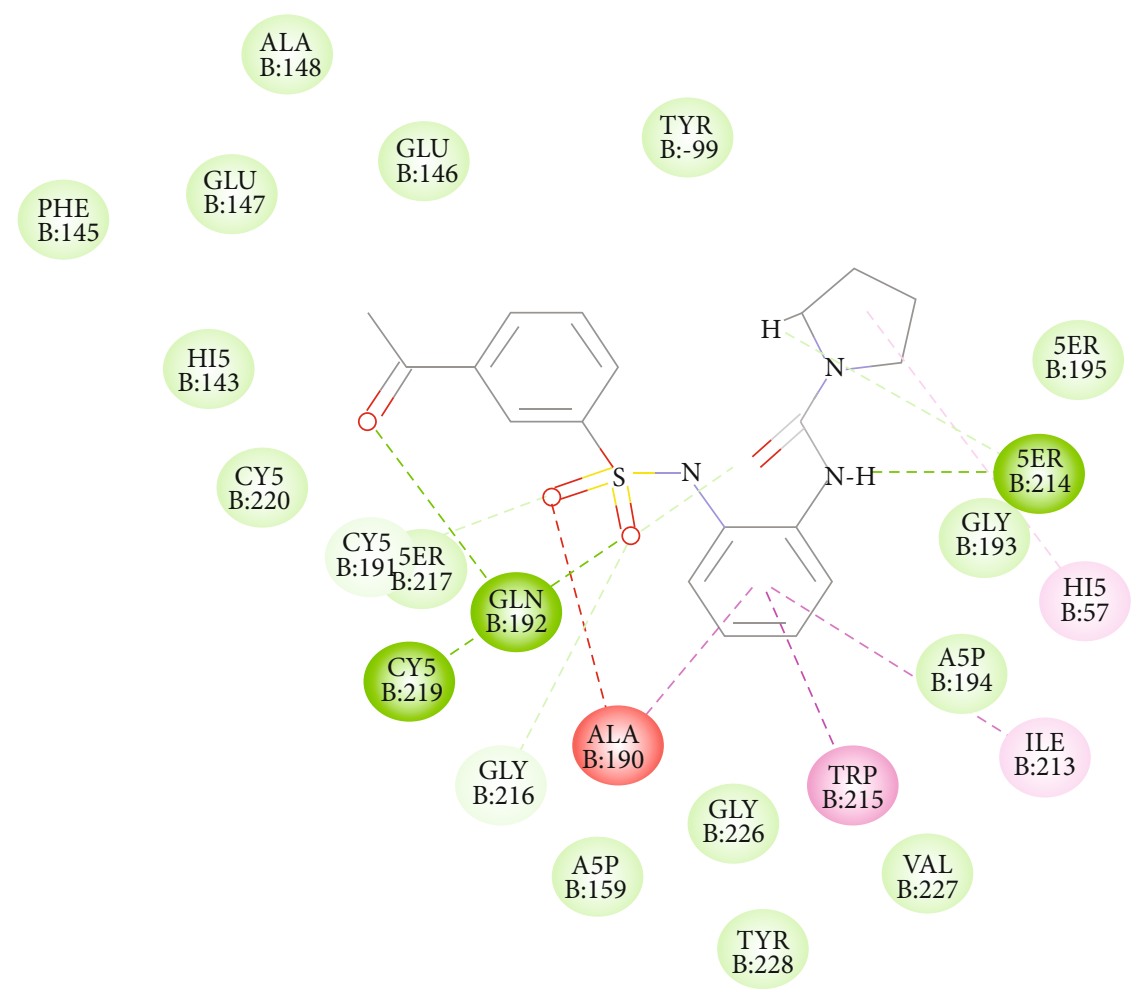

Interactions
$\square$ Van der Waals

Conventional hydrogen bond

Carbon hydrogen bond
Unfavorable acceptor-acceptor

Amide-Pi stacked

Pi-alkyl

(c)

FIGURE 2: Binding mode of Z432246974 to the FXIIa. (a) Overall structure of FXIIa-Z432246974 complex showing protein in cartoon model and ligand in stick. Showing the binding site residue surface around the ligand. Displaying the H-bond donor (purple) and acceptor (green) area. (b) Interactions of Z432246974 (sticks) to the FXIIa residues (sticks). (c) 2D diagram of FXIIa interactions to compound Z432246974.

is stabilized by forming noncovalent interaction between Z1225120358 and FXIIa residues.

In another complex, Z432246974 binds to the active site cavity of the FXIIa. The estimated binding affinity is also significantly high $(-36.396 \mathrm{kcal} / \mathrm{M})$ (Figure 2(a)). There are three hydrogen bond interactions observed (Gln192, Ser214, and Gly219) for Z432246974 to the FXIIa and many van der Waals interactions offered by Tyr99, Ala190, Glu146, Ile213, Gly147, Asp189, Cys220, Ser217, Gly226, Val227, and Tyr228 (Figures 1(b) and 1(c)). The complex of FXIIa- 


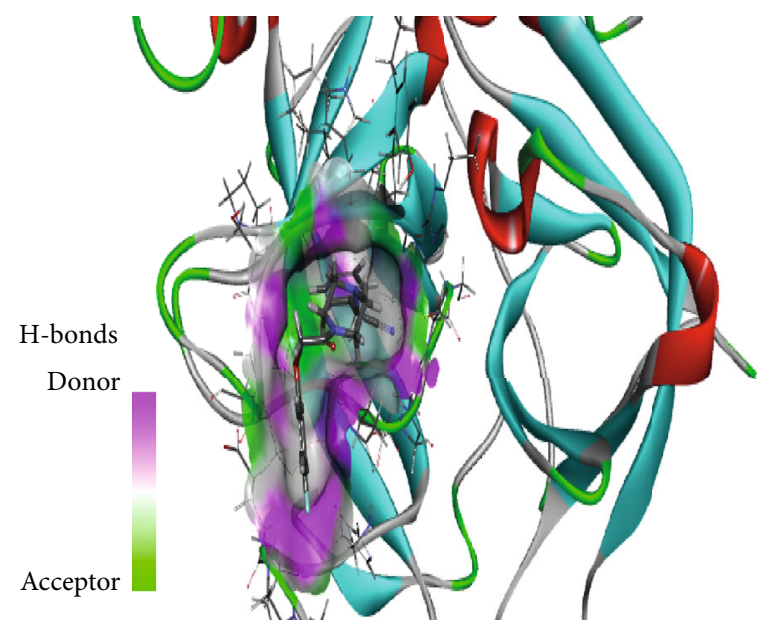

(a)

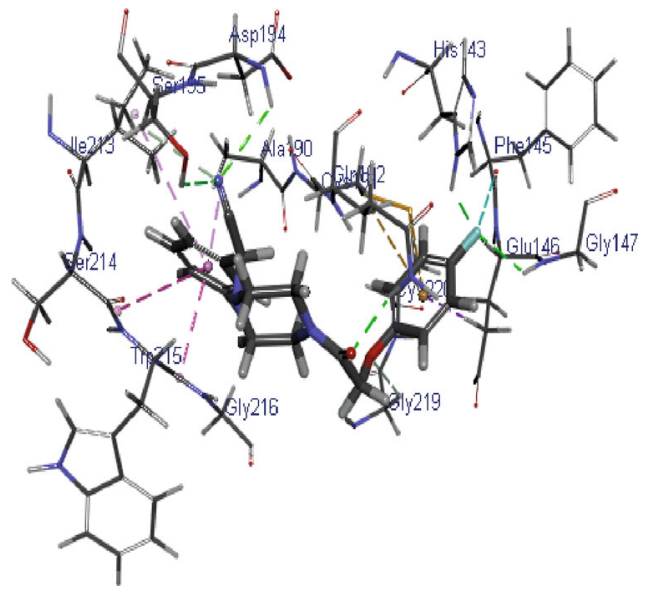

(b)

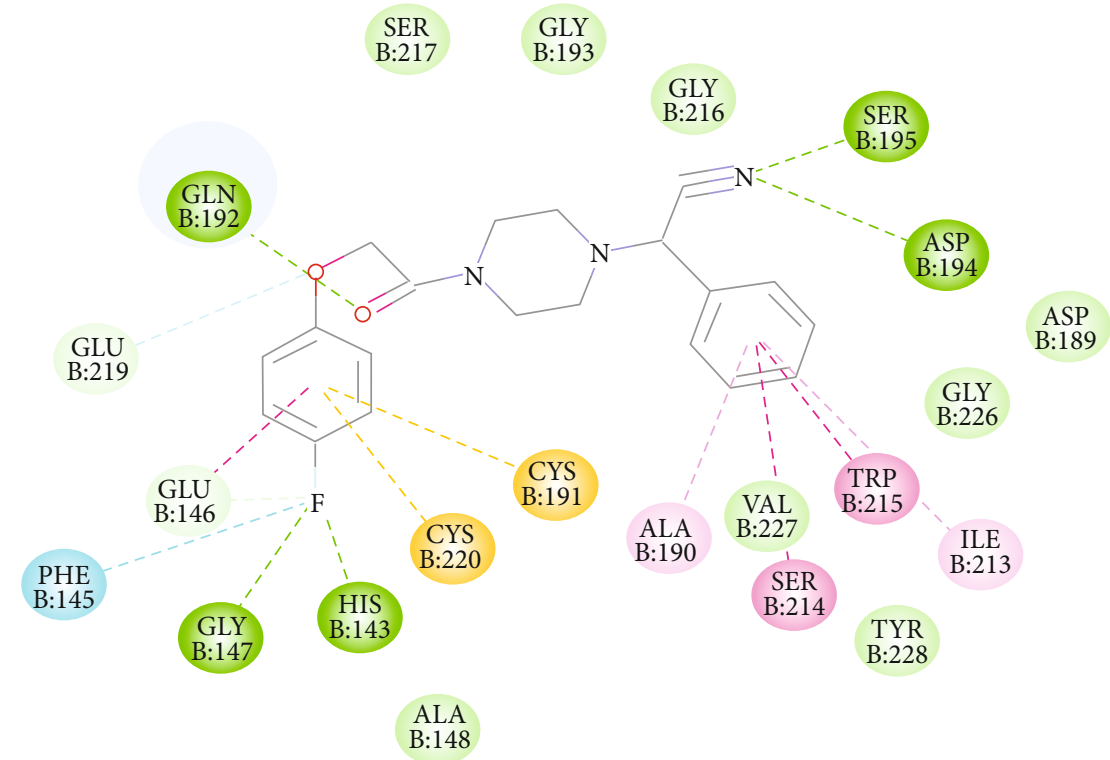

Interactions
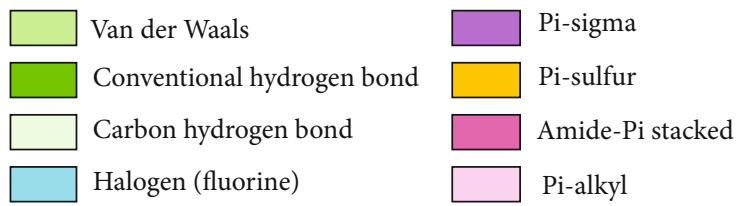

(c)

FIgURE 3: Binding mode of Z432246974 to the FXIIa. (a) Overall structure of FXIIa-Z146790068 complex showing protein in cartoon model and ligand in stick. Showing the binding site residue surface around the ligand. Displaying the H-bond donor (purple) and acceptor (green) area. (b) Interaction of Z146790068 (stick) to the FXIIa residues (stick). (c) 2D diagram of FXIIa interaction to compound Z146790068.

Z1225120358 is stabilized by forming several noncovalent interactions with FXIIa residues.

Similarly, Z146790068 also binds to the active site cavity of FXIIa with a binding affinity of $-25.434 \mathrm{kcal} / \mathrm{M}$ (Figure $3(\mathrm{a})$ ). There are five hydrogen bond interactions observed for Z146790068 to the FXIIa residues (His143, Gly147, Gln192, Asp194, and Ser195) and many van der Waals interactions offered by Phe145, Glu146, Ala190, Cys191, Ile213, Ser214, Trp215, Gly216, and Gly219 (Figures 3(b) and $3(c))$. The complex of FXIIa-Z146790068 is stabilized by the formation of several noncovalent interactions offered by FXIIa residues.

Compound Z45287215 showed the highest binding affinity of $-65.195 \mathrm{kcal} / \mathrm{mol}$, Three hydrogen bond interactions were observed (Gly147, Ser217, and Gly219) for Z45287215 to FXIIa, as well as many van der Waals interactions offered by Asp189, Ala190, Gln192, Ile213, Gly216, Ser217, Gly226, Val227, and Tyr228 (Figures 4(b) and 4(c)). The complex of FXIIa-Z45287215 is stabilized by forming noncovalent interaction between Z45287215 and FXIIa residues. 


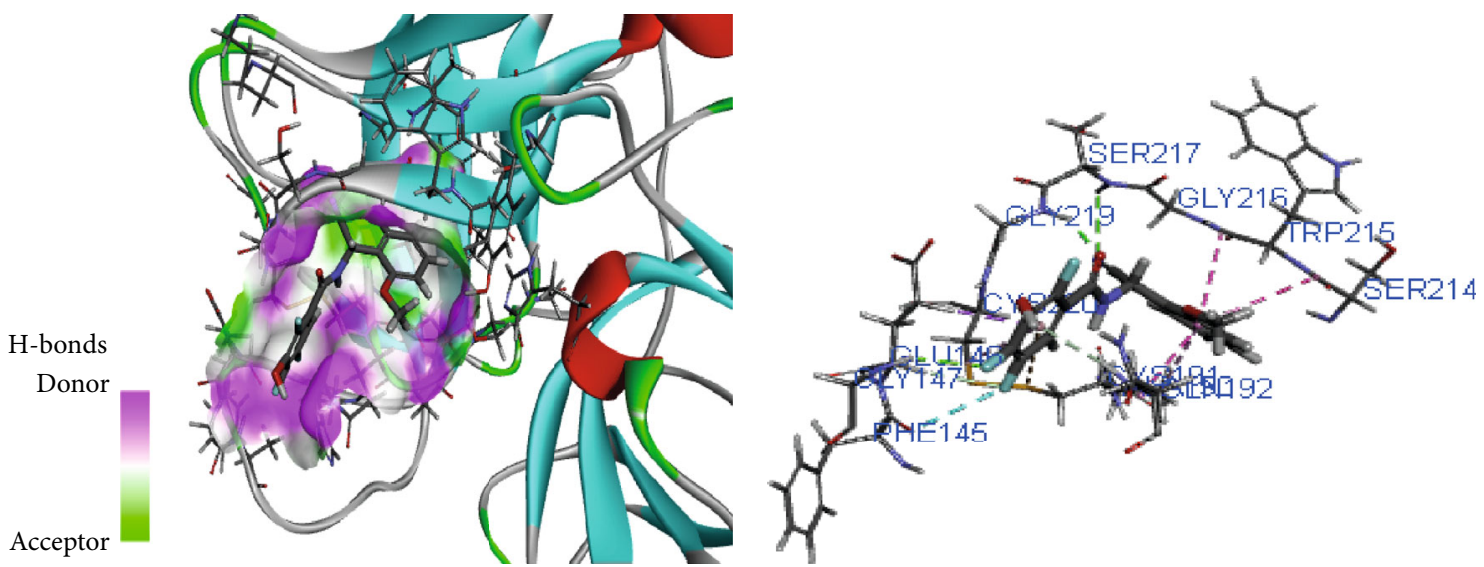

(a)

(b)

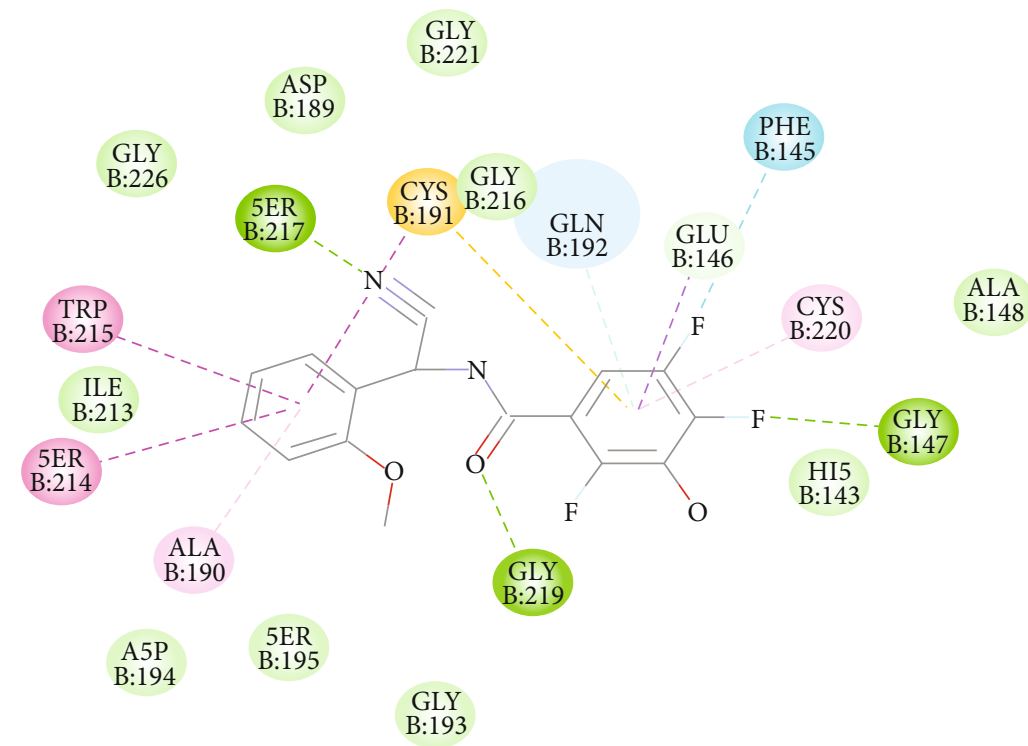

Interactions

Van der Waals

Conventional hydrogen bond

Carbon hydrogen bond

Halogen (fluorine)

Pi-donor hydrogen bond

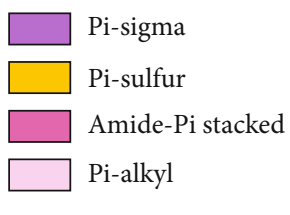

(c)

FIGURE 4: Binding mode of Z45287215 to the FXIIa. (a) Overall structure of FXIIa-Z45287215 complex showing protein in cartoon model and ligand in stick. Showing the binding site residue surface around the ligand. Displaying the H-bond donor (purple) and acceptor (green) area. (b) Interaction of Z45287215 (stick) to the FXIIa residues (stick). (c) 2D diagram of FXIIa interaction to compound Z45287215.

3.4. Molecular Dynamics Simulation. To further analyze protein-ligand interactions, we selected the top three ADMET compounds for molecular dynamics simulation. MD simulations were performed for FXIIa, FXIIa-Z1225120358 complex, FXIIa-Z432246974 complex, and FXIIa-Z146790068 complex by $200 \mathrm{ps}$. The constant temperature fluctuations at $300 \mathrm{~K}$ for each system suggest a stable and accurate nature of the MD simulations. The average potential energy of FXIIa, FXIIaZ1225120358 complex, FXIIa-Z432246974 complex, and FXIIa-Z146790068 complex was analyzed. An average potential energy for FXIIa, FXIIa-Z1225120358 complex, FXIIa-
Z432246974 complex, and FXIIa-Z146790068 complex was found to be $-75682 \mathrm{~kJ} / \mathrm{mol},-76531 \mathrm{~kJ} / \mathrm{mol},-76632 \mathrm{~kJ} / \mathrm{mol}$, and $-76594 \mathrm{~kJ} / \mathrm{mol}$, respectively.

The RMSD value is used to measure the structural alterations in MD simulation [37]. As seen in Figure 5(a), the RMSD values of each simulation-maintained fluctuations are all less than $1.1 \AA$. The average root mean square deviation (RMSD) values were found to be $1.064 \AA$, $0.963 \AA$, $1.076 \AA$, and $1.025 \AA$ for FXIIa, FXIIa-Z1225120358 complex, FXIIa-Z432246974 complex, and FXIIa-Z146790068 complex, respectively. The binding of Z1225120358 and 


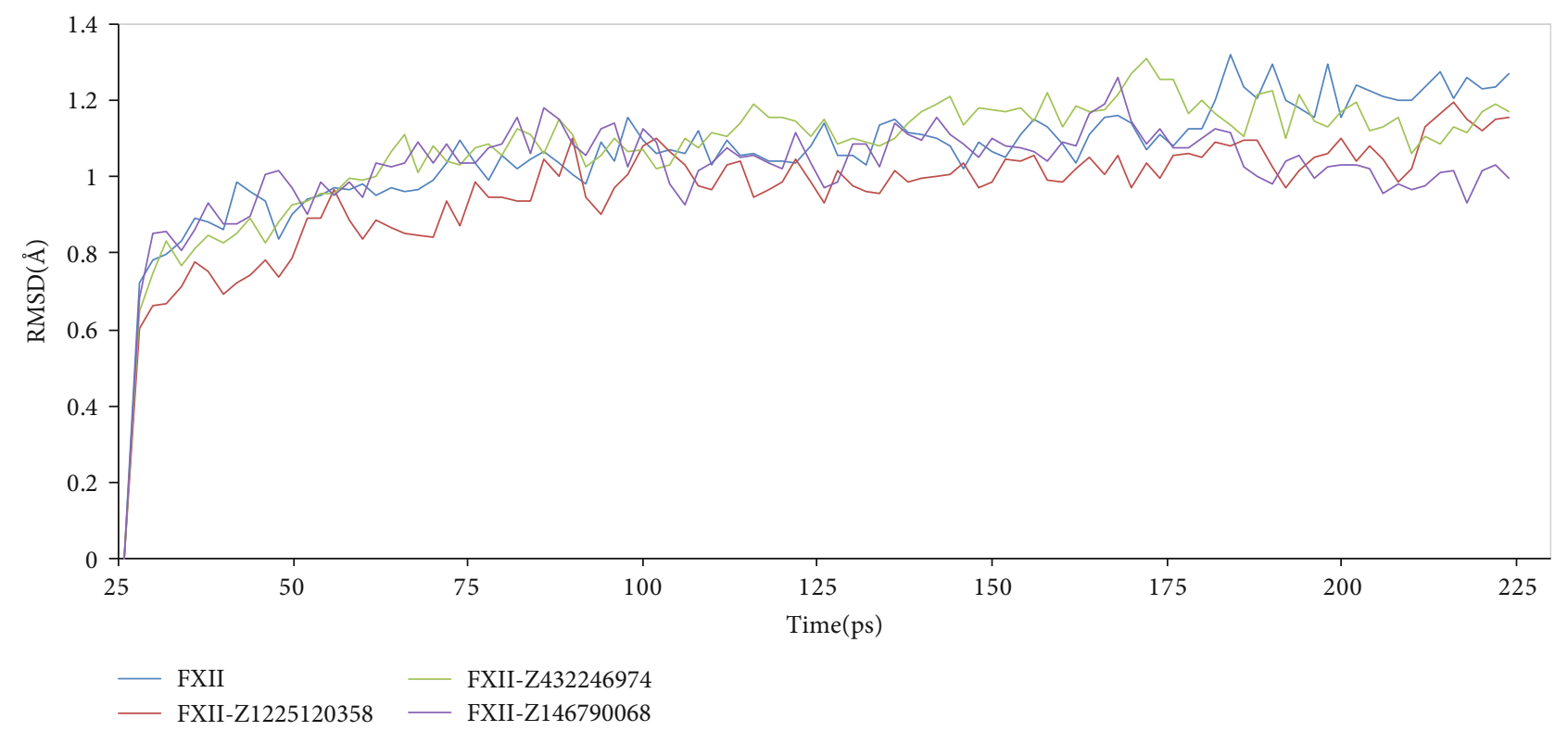

(a)

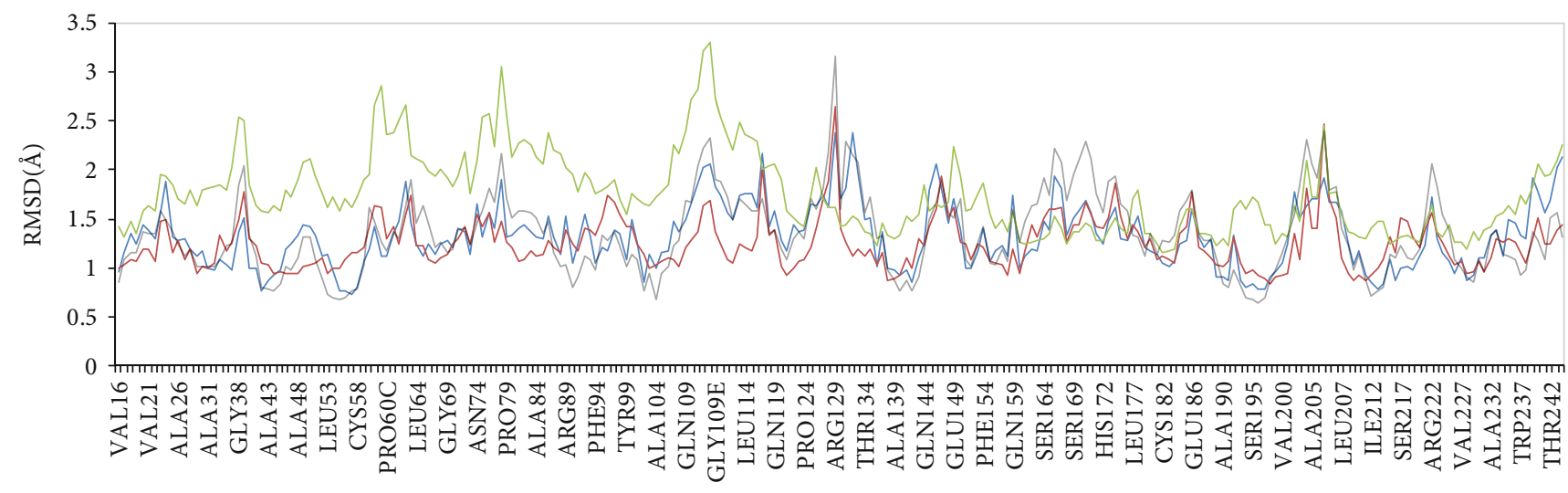

Residue

$\begin{array}{ll}- \text { FXII } & \text { FXII-Z432246974 } \\ \text { FXII-Z1225120358 } & - \text { FXII-Z146790068 }\end{array}$

(b)

Figure 5: RMSD values (a) and RMSF values (b) of FXIIa and its complexes with inhibitors as a function of time obtained for MD simulation.

Z146790068 to the FXIIa leads to a decrease in the RMSD values, whereas the binding of Z432246974 to the FXIIa leads to an increase of RMSD value. The results indicate that Z1225120358 and Z146790068 compounds tightly bind to the active pocket of FXIIa, whereas bound by compound Z432246974 leads to higher structural deviations of FXIIa. On this basis, fluctuations of FXIIa residues were declined and the activity of FXIIa may be inhibited by compounds Z1225120358 and Z146790068.

RMSF value is considered as the criterion of the overall flexibility in MD simulation [38, 39]. Moreover, we also investigated the motion of key residues of FXIIa interacted with inhibitors [35]. As shown in Figure 5(b), the RMSF values of FXIIa-Z1225120358 complex and FXIIa-Z146790068 complex are less than that of FXIIa, while the RMSF value for FXIIa-Z432246974 complex is greater.
Inhibition of activated factor XII (a-FXIIa) by five compounds Z1225120358, Z432246974, Z45287215, Z30974175, and Z146790068, with pIC50 values of $9.3 * 10^{-7}, 3.0 *$ $10^{-5}, 7.8 * 10^{-7}, 8.7 * 10^{-7}$, and $1.3 * 10^{-6} \mathrm{M}$, respectively (Figure 6, Table 3). The results showing that compounds Z1225120358, Z45287215, Z30974175, and Z146790068 could be more inhibition of FXIIa than Z432246974. The AMDET properties of Z45287215 and Z30974175 show not well but have better inhibition activity. The results of biological activity experiments combined with structural analysis. Three hydrogen bond interactions were observed (His143, Gly147, and Gly219) for Z1225120358 to FXIIa, and three hydrogen bond interactions were observed (Gly147, Ser217, and Gly219) for Z45287215 to FXIIa. His143, Ser217, Gly147, and Gly219 in the FXIIa specificity pocket play an important role in inhibiting FXII activity. 


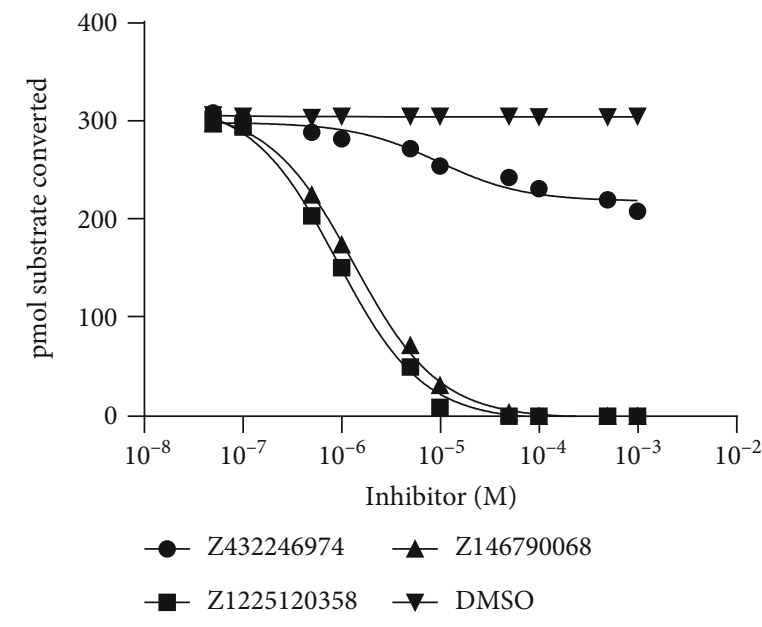

(a)

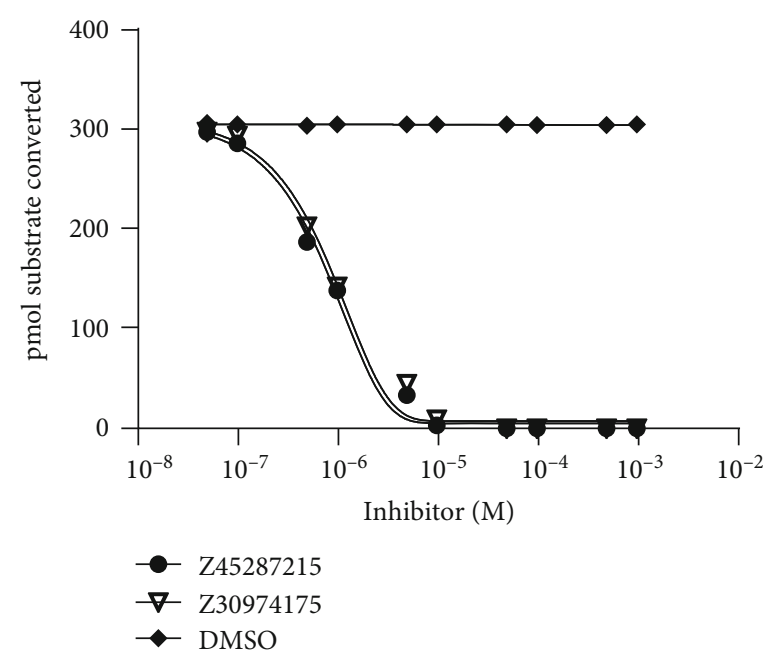

(b)

FIgURE 6: Inhibition of activated factor XII (a-FXIIa) by five compounds Z1225120358, Z45287215, Z30974175, Z432246974, and Z146790068. Concentrations of these five compounds $\left(10^{-8} \mathrm{M}\right.$ to $\left.10^{-3} \mathrm{M}\right)$ were incubated with $200 \mu \mathrm{M}$ substrate peptide, and this was followed by addition of a-FXIIa; enzymatic activity was then monitored as described in Materials and Methods. pIC50 values were obtained by nonlinear regression (GraphPad Prism V8.0.2.263; $\log [$ inhibitor] versus response-variable slope algorithm with a bottom constraint). Error bars indicate the standard error ( $n=3$ independent observations).

TABLE 3: Inhibition of activated factor XII (a-FXIIa) by five compounds.

\begin{tabular}{lccccc}
\hline Compounds & Z1225120358 & Z432246974 & Z45287215 & Z30974175 & Z146790068 \\
\hline pIC50 values $(M)$ & $9.3 * 10^{-7}$ & $3.0 * 10^{-5}$ & $7.8 * 10^{-7}$ & $8.7 * 10^{-7}$ & $1.3 * 10^{-6}$ \\
\hline
\end{tabular}

\section{Conclusions}

FXII is an emerging promising target for serious diseases. It plays important roles in thrombosis, hemostasis, and additional pathologic settings [40]. We have identified three potential inhibitors of FXIIa using HTS and MD simulation. All these three compounds bind to the common residues of the active site cavity of the FXIIa. High-throughput virtual screening was performed by a docking method. The top eighteen candidate inhibitors were further selected by AMDET prediction. Following MD simulation for FXIIa-ligand complexes effectively revealed that all of candidate compounds bind to the FXIIa active site. MD simulation results showed that bond with compound Z432246974 leads to higher structural deviations for FXIIa, suggesting that compound Z432246974 is not stable. We also found that compound Z1225120358 and Z146790068 could be more inhibition of FXIIa than Z432246974. Inhibition of activated factor XII assay and MD simulation, which had the same result, confirm that the analytical theory of MD simulation was correct. Inhibition of activated factor XII (a-FXIIa) by five compounds Z1225120358, Z432246974, Z45287215, Z30974175, and Z146790068, with pIC50 values of $9.3 * 10^{-7}, 3.0 *$ $10^{-5}, 7.8 * 10^{-7}, 8.7 * 10^{-7}$, and $1.3 * 10^{-6} \mathrm{M}$, respectively. The AMDET properties of Z45287215 and Z30974175 show not well but have better activity than Z1225120358. The results of biological activity experiments combined with structural analysis, His143, Ser217, Gly147, and Gly219 in the FXIIa specificity pocket, which play an important role in inhi- biting FXII activity. In conclusion, compounds Z1225120358, Z45287215, Z30974175, and Z146790068 were anticipated to be promising drug candidates for inhibition of FXIIa. Further experimental validations are required to confirm the inhibitory potential of these ligands against FXIIa.

\section{Abbreviations}

FXIIa: Human blood coagulation factor XIIa

MD: $\quad$ Molecular dynamics

HTS: $\quad$ High-throughput screening

ADMET: Absorption, distribution, metabolism, excretion, toxicity

RMSD: Root mean square deviation

RMSF: Root mean square fluctuation.

\section{Data Availability}

The underlying data in this manuscript were taken from the study by Zunyi Medical University and are cited in this study.

\section{Conflicts of Interest}

The authors declare that they have no conflicts of interest.

\section{Acknowledgments}

This work was supported by the National Natural Science Foundation of China under Grant (31660245), the Scientific 
Research Foundation for the Doctoral Scholars, Guizhou Department of Science and Technology under Grant ([2017]1224), and the Central Specialized Talent Foundation, Zunyi Medical University.

\section{References}

[1] T. Renné, A. H. Schmaier, K. F. Nickel, M. Blombäck, and C. Maas, "In vivo roles of factor XII," Blood, vol. 120, no. 22, pp. 4296-4303, 2012.

[2] R. W. Colman and A. H. Schmaier, "Contact system: a vascular biology modulator with anticoagulant, profibrinolytic, antiadhesive, and proinflammatory attributes," Blood, vol. 90, no. 10, pp. 3819-3843, 1997.

[3] C. Kannemeier, A. Shibamiya, F. Nakazawa et al., "Extracellular RNA constitutes a natural procoagulant cofactor in blood coagulation," Proceedings of the National Academy of Sciences of the United States of America, vol. 104, no. 15, pp. 6388-6393, 2007.

[4] J. M. Koenig, A. Chahine, and O. D. Ratnoff, "Inhibition of the activation of Hageman factor (factor XII) by soluble human placental collagens types III, IV, and V," Journal of Laboratory and Clinical Medicine, vol. 117, no. 6, pp. 523-527, 1991.

[5] M. Samuel, R. A. Pixley, M. A. Villanueva, R. W. Colman, and G. B. Villanueva, "Human factor-xii (Hageman-factor) autoactivation by dextran sulfate - circular-dichroism, fluorescence, and ultraviolet difference spectroscopic studies," Journal of Biological Chemistry, vol. 267, no. 27, pp. 19691-19697, 1992.

[6] J. Emsley, P. McEwan, and D. Gailani, "Structure and function of factor XI," Blood, vol. 115, no. 13, pp. 2569-2577, 2010.

[7] A. Dementiev, A. Silva, C. Yee et al., "Structures of human plasma $\beta$-factor XIIa cocrystallized with potent inhibitors," Blood Advances, vol. 2, no. 5, pp. 549-558, 2018.

[8] M. L. von Brühl, K. Stark, A. Steinhart et al., "Monocytes, neutrophils, and platelets cooperate to initiate and propagate venous thrombosis in mice in vivo," Journal of Experimental Medicine, vol. 209, no. 4, pp. 819-835, 2012.

[9] M. Pathak, P. Wilmann, J. Awford et al., "Coagulation factor XII protease domain crystal structure," Journal of Thrombosis and Haemostasis, vol. 13, no. 4, pp. 580-591, 2015.

[10] F. Müller, D. Gailani, and T. Renné, "Factor XI and XII as antithrombotic targets," Current Opinion in Hematology, vol. 18, no. 5, pp. 349-355, 2011.

[11] T. Renné, M. Pozgajová, S. Grüner et al., "Defective thrombus formation in mice lacking coagulation factor XII," Journal of Experimental Medicine, vol. 202, no. 2, pp. 271-281, 2005.

[12] C. Kleinschnitz, G. Stoll, M. Bendszus et al., "Targeting coagulation factor XII provides protection from pathological thrombosis in cerebral ischemia without interfering with hemostasis," Journal of Experimental Medicine, vol. 203, no. 3, pp. 513-518, 2006.

[13] A. Matafonov, P. Y. Leung, A. E. Gailani et al., "Factor XII inhibition reduces thrombus formation in a primate thrombosis model," Blood, vol. 123, no. 11, pp. 1739-1746, 2014.

[14] N. S. Key, "Epidemiologic and clinical data linking factors XI and XII to thrombosis," Hematology, vol. 2014, no. 1, pp. 6670, 2014.

[15] S. J. Middendorp, J. Wilbs, C. Quarroz, S. Calzavarini, A. Angelillo-Scherrer, and C. Heinis, "Peptide macrocycle inhibitor of coagulation factor XII with subnanomolar affinity and high target selectivity," Journal of Medicinal Chemistry, vol. 60, no. 3, pp. 1151-1158, 2017.

[16] C. M. Barbieri, X. Wang, W. Wu et al., "Factor XIIa as a novel target for thrombosis: target engagement requirement and efficacy in a rabbit model of microembolic signals," Journal of Pharmacology and Experimental Therapeutics, vol. 360, no. 3, pp. 466-475, 2017.

[17] M. Larsson, V. Rayzman, M. W. Nolte et al., "A factor XIIa inhibitory antibody provides thromboprotection in extracorporeal circulation without increasing bleeding risk," Science Translational Medicine, vol. 6, no. 222, article 222ra17, 2014.

[18] I. Hagedorn, S. Schmidbauer, I. Pleines et al., "Factor XIIa inhibitor recombinant human albumin infestin-4 abolishes occlusive arterial thrombus formation without affecting bleeding," Circulation, vol. 121, no. 13, pp. 1510-1517, 2010.

[19] J. W. Yau, A. R. Stafford, P. Liao et al., "Corn trypsin inhibitor coating attenuates the prothrombotic properties of catheters in vitro and in vivo," Acta Biomaterialia, vol. 8, no. 11, pp. 4092-4100, 2012.

[20] V. Baeriswyl, S. Calzavarini, S. Chen et al., "A synthetic factor XIIa inhibitor blocks selectively intrinsic coagulation initiation," ACS Chemical Biology, vol. 10, no. 8, pp. 1861-1870, 2015.

[21] R. S. Woodruff, Y. Xu, J. Layzer, W. Wu, M. L. Ogletree, and B. A. Sullenger, "Inhibiting the intrinsic pathway of coagulation with a factor XII-targeting RNA aptamer," Journal of Thrombosis and Haemostasis, vol. 11, no. 7, pp. 1364-1373, 2013.

[22] T. Q. Cai, W. Wu, M. K. Shin et al., "Factor XII full and partial null in rat confers robust antithrombotic efficacy with no bleeding," Blood Coagulation \& Fibrinolysis, vol. 26, no. 8, pp. 893-902, 2015.

[23] A. S. Revenko, D. Gao, J. R. Crosby et al., "Selective depletion of plasma prekallikrein or coagulation factor XII inhibits thrombosis in mice without increased risk of bleeding," Blood, vol. 118, no. 19, pp. 5302-5311, 2011.

[24] J. I. Weitz, "Factor Xa and thrombin as targets for new oral anticoagulants," Thrombosis Research, vol. 127, pp. S5-S12, 2011.

[25] K. M. Merz Jr., Drug Design: Structure- and Ligand-Based Approaches, D. Ringe and C. H. Reynolds, Eds., Cambridge University Press, Cambridge, 2010.

[26] M. I. Hassan, V. Kumar, R. K. Somvanshi, S. Dey, T. P. Singh, and S. Yadav, "Structure-guided design of peptidic ligand for human prostate specific antigen," Journal of Peptide Science, vol. 13, no. 12, pp. 849-855, 2007.

[27] H. Naz, E. Jameel, N. Hoda et al., "Structure guided design of potential inhibitors of human calcium-calmodulin dependent protein kinase IV containing pyrimidine scaffold," Bioorganic \& Medicinal Chemistry Letters, vol. 26, no. 3, pp. 782-788, 2016.

[28] B. R. Brooks, C. L. Brooks 3rd, Mackerell AD Jr et al., "CHARMM: the biomolecular simulation program," Journal of Computational Chemistry, vol. 30, no. 10, pp. 1545-1614, 2009.

[29] J. Tirado-Rives and W. L. Jorgensen, "Contribution of conformer focusing to the uncertainty in predicting free energies for protein-ligand binding," Journal of Medicinal Chemistry, vol. 49, no. 20, pp. 5880-5884, 2006.

[30] M. L. Zhao, W. Wang, H. Nie, S. S. Cao, and L. F. du, "In silico structure prediction and inhibition mechanism studies of AtHDA14 as revealed by homology modeling, docking, 
molecular dynamics simulation," Computational Biology and Chemistry, vol. 75, pp. 120-130, 2018.

[31] C. H. Lin, T. T. Chang, M. F. Sun et al., "Potent inhibitor design against $\mathrm{H} 1 \mathrm{~N} 1$ swine influenza: structure-based and molecular dynamics analysis for M2 inhibitors from traditional Chinese medicine database," Journal of Biomolecular Structure \& Dynamics, vol. 28, no. 4, pp. 471-482, 2011.

[32] F. R. Makhouri and J. B. Ghasemi, "High-throughput docking and molecular dynamics simulations towards the identification of novel peptidomimetic inhibitors against CDC7," Molecular Informatics, vol. 37, no. 11, 2018.

[33] A. Beg, F. I. Khan, K. A. Lobb, A. Islam, F. Ahmad, and M. I. Hassan, "High throughput screening, docking and molecular dynamics studies to identify potential inhibitors of human calcium/calmodulin-dependent protein kinase IV," Journal of Biomolecular Structure and Dynamics, vol. 37, no. 8, pp. 2179-2192, 2018.

[34] A. N. Singh, M. M. Baruah, and N. Sharma, "Structure based docking studies towards exploring potential anti-androgen activity of selected phytochemicals against prostate cancer," Scientific Reports, vol. 7, no. 1, article 1955, 2017.

[35] S. Zang, G. Dong, B. Peng et al., "A comparison of physicochemical properties of sterilized chitosan hydrogel and its applicability in a canine model of periodontal regeneration," Carbohydrate Polymers, vol. 113, pp. 240-248, 2014.

[36] M. R. Feng, "Assessment of blood-brain barrier penetration: in silico, in vitro and in vivo," Current Drug Metabolism, vol. 3, no. 6, pp. 647-657, 2002.

[37] R. J. Hyndman and A. B. Koehler, "Another look at measures of forecast accuracy," International Journal of Forecasting, vol. 22, no. 4, pp. 679-688, 2006.

[38] W. Wang, X. Li, Q. Wang, X. Zhu, Q. Zhang, and L. du, “The acidic $\mathrm{pH}$-induced structural changes in apo-CP43 by spectral methodologies and molecular dynamics simulations," Journal of Molecular Structure, vol. 1152, pp. 177-188, 2018.

[39] L. Xi, Y. Wang, Q. He, Q. Zhang, and L. du, "Interaction between Pin1 and its natural product inhibitor epigallocatechin-3-gallate by spectroscopy and molecular dynamics simulations," Molecular and Biomolecular Spectroscopy, vol. 169, pp. 134-143, 2016.

[40] K. F. Nickel, A. T. Long, T. A. Fuchs, L. M. Butler, and T. Renné, "Factor XII as a therapeutic target in thromboembolic and inflammatory diseases," Arteriosclerosis Thrombosis and Vascular Biology, vol. 37, no. 1, pp. 13-20, 2017. 